\title{
A conversation with Thomas Südhof
}

Thesi he simple fact that you can understand the words on this page, and that you might remember any of this interview tomorrow, is thanks to the simultaneous and precise coordination of communication between the billions of neurons in your brain and peripheral nervous system. Herein, the JCI speaks with Thomas Südhof (Figure 1) of Stanford University, who has been at the center of unlocking the secrets of neurotransmission. His work over the last 30 years has elucidated much of what we know about the molecular mechanisms of neurotransmission in synaptic signaling. For this body of work, Südhof has recently shared in the 2013 Nobel Prize in Physiology or Medicine and the 2013 Albert Lasker Basic Medical Research Award. The full interview can be seen on the JCI website, http://www.jci.org/kiosk/cgm.

JCI: Can you tell me a little bit about your upbringing?

Südhof: I was born and grew up in Germany. I was actually born in Göttingen, which is a small university city very close to Hanover. My parents were both physicians and my father was at the university. He was interested in how diseases develop in terms of metabolism and, in particular in lipid metabolism, which is kind of ironic, given my later career. I went through the regular schooling in Germany. And while I was a teenager my father died, so that certainly influenced my development considerably. After school I decided to study medicine and to become a physician myself, not because of the family tradition, there was none apart from my own parents, but because I felt that medicine was the best way to learn as much as possible about the world and to be in a later position to do something practical and useful.

JCI: As a child, were you fascinated by science?

Südhof: To be honest, no. When I was brought up I had a very broad background that included a lot of humanistic types of learning including classical music, literature, and visual arts, as well as a broad background in natural sciences. I didn't become interested in science until I was in medical school, and I realized that our ways of approaching disease, of trying to help people who are sick, of trying to be of some help to anybody depends on an understanding of the fundamental facts.
My career path was a little unusual. I finished medical school, I went through an internship, and afterwards I actually had intended to pursue an academic career in Germany. I was particularly interested in clinical medicine. An academic career in Germany in clinical medicine requires scientific training and I decided to obtain that scientific training beyond my doctoral degree by coming to the States. I did this with the full desire to go back and complete a full residency and become a doctor.

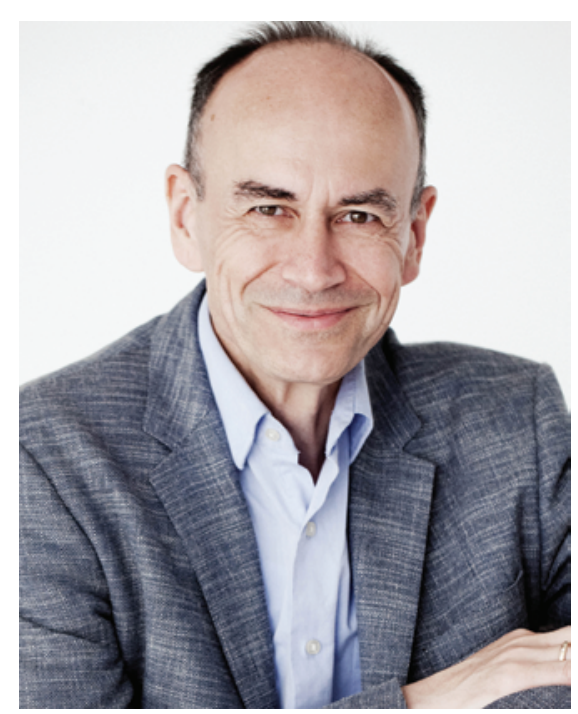

Figure 1

Thomas Südhof on September 19, 2013. Image credit: Alena Soboleva.

JCI: Your doctoral lab research was working on chromaffin cells with Victor Whittaker.

Südhof: I looked for laboratory I could work in Göttingen, where I was. Göttingen had a very nice Max Planck Institute, founded by Manfred Eigen, a Nobel Prize winning chemist. Victor Whittaker, who was a director at the Institute said, "Alright, you can work here, you can work with this assistant I have - this sort of most senior scientist who is a permanent member of my staff," and so I started working with that person. Things were pretty flexible then and I worked there, often at night, and then the person I was supposed to work with left after half a year or so. I was pretty much left to my own devices, with a lot of advice from Victor Whittaker, but no real mentor who would be there on a daily basis. That freedom was actually extremely inspiring because it allowed me to try all kinds of things. I decided to work on chromaffin cells and specifically on chromaffin granules, which were the secretory vesicles of these cells because I had learned from others in the Institute that was a source where you can purify an organelle and study the organelle. I thought that we don't know anything about the molecular and biophysical properties of organelles and so I might as well study that.

JCI: How did you land in the Brown and Goldstein lab for your postdoc?

Südhof: As I was going through my internship, I decided that I wanted to be an academic clinician. In order to be an academic clinician, to get scientific training I needed to get the best possible training in what seemed to me to be the most interesting advances or fields at that time. Others made me aware of Mike Brown and Joe Goldstein's excellent work and at that point they were perhaps not as famous as they are now.

They were in Dallas, Texas, which wasn't exactly a let's say, famous center of science, so it wasn't necessarily an obvious choice. In fact, I myself was somewhat hesitant because of the location. But I went to Dallas to visit and interview, and I think everybody in the world would have been incredibly impressed. And that's when I decided to apply, and I was extremely happy that I was invited to join their lab.

JCI: You fell into a project wherein you cloned the LDL receptor gene.

Südhof: I arrived in October ' 83 - a very exciting time in science because molecular biology had basically been invented in the previous decade. I became completely immersed in cholesterol metabolism and receptor biology. The whole field of LDL and cholesterol revolved around the metabolic pathways that cholesterol was involved in. What is nowadays textbook knowledge was completely unknown. The whole notion that you have cell surface receptors that recycle and that are regulated, that bind things and take them up, and that this is a vital component of every cell's existence.

In my three years in Mike and Joe's lab, I worked on two related projects, the cloning of the LDL receptor gene and something that had even more long-term repercussions: the identification of sequence 
elements in the LDL receptor gene that mediated the regulation of the expression of the LDL receptor gene by cholesterol itself. This whole mechanism of how cholesterol regulates gene expression then led to the discovery by Mike and Joe's lab of the whole cholesterol-dependent regulatory transcription pathway - the SREBP pathway and so on.

JCI: Given your excitement surrounding SREBPs and LDL, why move on to synaptic biology?

Südhof: There are many answers to this question. Nowadays, virtually nobody dares to change fields, especially during the transition from a postdoc to independent lab head. People are afraid of not having enough funding. But in biology, I think there's a big danger of becoming a specialist in one little area, which stifles creativity and really is not necessarily a good longterm career move because you never learn anything new. You only learn new techniques, but you don't actually learn new concepts. I still think that even though techniques are often rewarded more prominently than conceptual advances, it is the conceptual advances that make science worth doing.

If you work next to two giants like Mike and Joe and you become independent, it is very difficult to have any real, completely independent ideas, thoughts, or developments if you work on the same field. So, my decision to change fields was a conscious decision in part, because I felt that if I was going to be independent, I wanted to do something that nobody else had done before.

JCI: Why synaptic neurotransmission?

Südhof: The most important event that happens in the brain is synaptic transmission, and the reason is that any communication between neurons, any information processing happens at synapses. Neural networks are formed by synapses. Synapses not only transmit information, they also compute it. Because they change, they have an individual memory and they change as a function of use. They are the most fundamental information-processing unit, not only in information relay stations in the brain. When I started, nothing was known about synapses, except that they communicated via the release of a chemical transmitter that then was recognized by postsynaptic receptors. I decided to start working on this problem in molecular terms, because not a single molecule had been cloned prior to this, not a single protein had been identified in molecular terms, and it seemed to me that understanding how this works would have tremendous effects on our understanding on how the brain works.

JCI: Your work over the years has focused largely on the presynaptic synapse. Why the pre-side and not the post-side?

Südhof: We initially started on the presynaptic side because it was more amenable to molecular dissection. We started by purifying synaptic vesicles, often in collaboration with my long-term collaborator, Reinhard Jahn, and then tried to understand the components. That was possible because of prior work that established how synaptic vesicles could be purified. Postsynaptically, not as much was known. However, during the time of this work, everything changed in the sense that the postsynaptic side became much easier to analyze due to the advance of electrophysiological technology, and because you can detect postsynaptic events much better than presynaptic events.

I started initially presynaptic because that was, at that point, technically the better avenue. I still work on presynaptic mechanisms because, after all, a neurotransmitter is what initiates synaptic transmission and is an absolutely essential part of synaptic transmission.

JCI: Synaptobrevin, synaptotagmin, RIM, Munc18, and recently neurexin and neuroligin. You've spent your career elucidating exactly what the mechanism of neurotransmission is and the proteins that are in the synapse. How have you managed to have this singular focus?

Südhof: My focus on synapses is driven by my continued belief that understanding synaptic transmission is absolutely crucial for understanding any brain function high level/low level memory, immediate reactions, vision, perception, thought, consciousness. A synapse is obviously going to be complicated. So, how can we actually deal with the complexity and the many components? One way to do this is to try to identify what components are there. Then, subsequently try to figure out which ones of these many components are the ones that actually matter; and which ones are the ones that are not going to provide major insights, which ones are the ones that are going to have real consequences for our understanding, and then focus on these components.

Among the list of proteins you mentioned, all of these turned out to be proteins that are of absolutely central importance. But there are many others I have studied over the years that you didn't men- tion, that I spent many years and a lot of papers on. And that, to this date, don't really have a well-known function, and probably are important in some way, but are not central players.

JCI: The elucidation of the biology of the synapse and other insights into membrane dynamics happened from several groups, often at the same time. These groups include Richard Scheller, Jim Rothman, and Randy Schekman. Was it a competitive landscape or a collaborative landscape?

Südhof: It was and still is a pretty competitive landscape, but the approaches were slightly different. Richard Scheller and I pursued quite similar approaches, but our proteins rarely overlapped. Where the most competition came in was probably in the discovery of how clostridial, tetanus, and botulinum toxins act. I don't think there was any competition in terms of the active zone as far as I can remember. It is now a tremendously competitive field, but it wasn't when I started this research 20 years ago.

JCI: In looking through your CV, most of your articles have tended to have fairly short author lists even in this growing age where author lists can reach into the hundreds. Is this part of your approach to mentoring, to work with smaller groups of scientists on focused ideas?

Südhof: I think that this is a complete reflection of the approach. I think team science is terrific because it enables doing things in a systematic manner that would otherwise not be possible. For example, genomics, huge expression studies, probably connectomics that is understanding of the anatomical basis of neurocircuits. Those kinds of approaches require large numbers of people working together, and the outcomes of these approaches are really important values that will be accessible and so on, forever, presumably. However, my science, my interest, is really to understand how synapses work and that doesn't require a team approach.

JCI: If you had to do it all over again would you have picked the same path?

Südhof: The culture I grew up in put an enormous value on the intrinsic importance of knowledge and truth, and I feel that has become devalued over the last decades. I think that I would be interested in an advocacy role, because I feel that, although there's much we don't know, we also must discuss, understand, promote, and ask new questions.

\section{Ushma S. Neill}

